\title{
THEORY OF GAMMA RAY BURSTERS
}

\author{
G.S.Bisnovatyi-Kogan \\ Space Research Institute \\ Acad. Sci. USSR, Moscow, \\ Profsoyuznaja 84/32, \\ USSR
}

\begin{abstract}
Gamma ray bursters are interpreted as nuclear explosions under the surface of neutron stars. The explosions occur after transportation of the matter with nonequilibrium composition during starquakes in outer layers where the matter becomes unstable and explodes as a result of a developing chain reaction. In the nonequilibrium matter nuclear charge $z$ decreases with increasing density, contrary to the equilibrium composition. Formation of a shock wave and total energy output are calculated. Parameters of the mighty burst of 5 March 1979 are estimated.Formation of observed lines in different partions of the $\gamma-$ range and a possible nature of accompanying optical bursts are discussed with the proposed model as a basis.
\end{abstract}

\section{INTRODUCTION}

Soon after the discovery of gamma ray bursters [1] about ten very different theories have been proposed for their explanation 3 . The discovery of a pulsar stage in the gamma ray burst of 5 March 1979 [3] was a stromg support to the theory related to neutron stars $[4,5]$. Intensive investigations of gamma-ray bursters in late $70 \mathrm{~s}-$ early $80 \mathrm{~s}$ gave vast information about complex temporal and spectral behaviour of gamma-ray bursts, their statistical properties connected with the log $N$ - log $s$ diagram, exact 10calization on the sky, etc. [6-9] . Despite numerous attempts of optical identification no burster have ever been associated with any stationary optical source $[7,8]$. Neither radio nor X-ray sources have been found in the error boxes of several well-localized sources $)^{3}$. In this indicates that the neutron stars, responsible for gamma-ray

*) The projection [7] of the 5 March 1979 burster on the edge of SNR N 49 in IMC seems accidental. 
bursts are single and very old with moderate ( $\leqslant \leqslant 10^{10} \mathrm{Gs}$ ) magnetic fields. The model of bursts from such objects has been developed in $[4,5,10-14]$. It is necessary in this model that a neutron star have a nonequilibrium layer of superheavy elements, stable at high densities and unstable at low ones. The transfer of the nonequilibrium matter to low densities must occur during starquakes which are often observed in young neutron stars (Crab and Vela pulsars) and may be very rare in old ones. This may explain almost total absence of recurrence in gamma bursters.

It is impossible now to explain with only one theoretical model all striking features of gamma-ray bursters. We try here to fit only the most important features: the energy supply, short rising tiffestrincluding formation of $\gamma$ lines, emission in hard and soft'regions. We give quantitative estimations of this characteristics and several observational predictions. The construction of the model seems to be a hard job, but it could have been enormousIy harder if the connection with the neutron stars had not been firmly established.

\section{FORMATION OF A NONEQUIIIBRIUM LAYER AND ITS STRUCTURE}

The dense matter may have an Equilibrium Composition only at high temperatures when all possible channels of reactions are open. The channels become shut during cooling so that strictiy speaking, the equilibrium in the cold matter (minimum energy state) is impossible. The cold matter composition is always nonequilibrium and depends on the

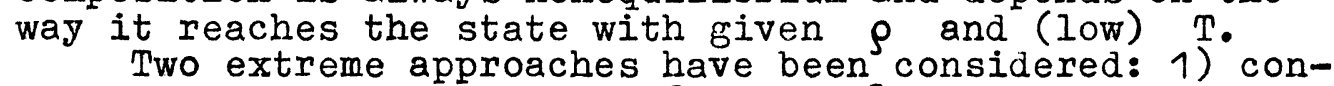
traction of the cold matter $[10,15-18]$ which occurs during the accretion on a neutron star; 2) cooling of the matter at a given density, which occurs after the neutron star is formed.

The contraction of cold matter continues with $A=$ const and decreasing $Z$ up to the neutron drip line where the stripping energy of the neutron is $Q_{n}=0$.

Further the contraction leads to cold evaporation of neutrons and decreasing both $A$ and $Z$ along the neutron drip line. Using nuclear parameters from [19] and beginning the compression framiron $F e^{56}$ we obtain the nucleus

$(A, Z)=(56,16)$ at $\rho=4,2 \cdot 10^{11} \mathrm{~g} / \mathrm{cm}^{3}$ on the neutron drip line. Calculations [18] based on the nuclear matter theory from [20] give nuclei along the neutron drip line

from $(A, Z)=(56,16)$ at $\rho=5,0110^{11} \mathrm{~g} / \mathrm{cm}^{3}$ up to 
$(A, Z)=(32,9)$ at $\rho=1,41 \cdot 10^{12} \mathrm{~g} / \mathrm{cm}^{3}$. As estimated in [18], further contration leads to fusion of the nuclei in picnonuclesr reactions.

Analytical estimations of parameters along the neutron drip line were made in $[15,16]$. The stripping energy of the proton $\mathrm{Q}_{\mathrm{p}}$ :

$$
Q_{p}(A, Z)=\left(m_{A, Z-1}-m_{A, Z}\right) C^{2}+Q_{n}(A, Z-1)-\left(m_{n}-m_{p}\right) C^{2}
$$

is approximated along the neutron arip line by relation

$$
Q_{p}=33-Z / 7 \mathrm{Mev}, A=4 Z, \quad Z \geqslant 6 .
$$

Using consition of beta-equilibrium

$$
\left(m_{A, Z-1}-m_{A, Z}\right) c^{2}=\varepsilon_{f e}+m_{e} c^{2}
$$

and expression for Fermi-energy $\varepsilon_{f e}$, we obtain from $(1)-(3)$ the relation between $z$ and $\rho$ at $Q_{n}=0$ :

$$
\left[1+\left(\frac{Z \rho}{10^{6} A_{0}}\right)^{2 / 3}\right]^{1 / 2}=1.96\left(33-\frac{Z}{7}\right)+2.54, A=4 Z
$$

The compositions along the neutron drip line from (4) are: $(A, Z)=(56,14)$ at $\rho=10^{12} \mathrm{~g} / \mathrm{cm}^{3}$ up to $(A, Z)=(24,6)$ at $\rho=2 \cdot 6 \cdot 10^{12} \mathrm{~g} / \mathrm{cm}^{3}$.

When cooling proceeds at a constant density much higher $A$ and $Z$ values may be reached $[15,16]$. They depend on the free-neutron concentration which may be found from the calculations of the kinetic of cooling. Such calculations have not been made, so we suppose that the mass concentration of neutrons is $X_{n}=1 / 2$. The final composition along the neutron drip line, using (1)-(3), is $[15,16]$ : 


$$
\begin{aligned}
Z & =7\left\{33-0.511\left[\left(\frac{\rho}{\mu_{\dot{Z}} 10^{6}}\right)^{2 / 3}+1\right]^{1 / 2}+1.293\right\}, \\
\mu_{Z} & =A / Z\left(1-X_{n}\right) ; A=4 Z
\end{aligned}
$$

The curve $\mathrm{Z}(\rho)$ for $\mathrm{X}_{\mathrm{q}}=1 / 2$ is presented in Fig.1. The value $Q_{p, \text { max }}$ is reached for $C_{24}$ when $\rho=\rho_{2}=$ $2.24 \cdot 10^{12} \mathrm{~g} / \mathrm{cm}^{3}$. At $\rho=\rho_{1}=1.210^{11} \mathrm{~g} / \mathrm{cm}^{3}$, when $\mathrm{Z}=150$ we have $\mathrm{Z}^{2} / \mathrm{A}=\mathrm{Z} / 4=37.5$ with the fission time $\tau_{\rho}=3 \cdot 10^{7}$ years. At densities $\rho<\rho_{1}$ and $\rho>\rho_{2}$ new seed nuclei are formed and matter becomes almost equilibrium. How the nonequilibrium composition forms during cooling is schematically in shown the Fig. 2 from [16].

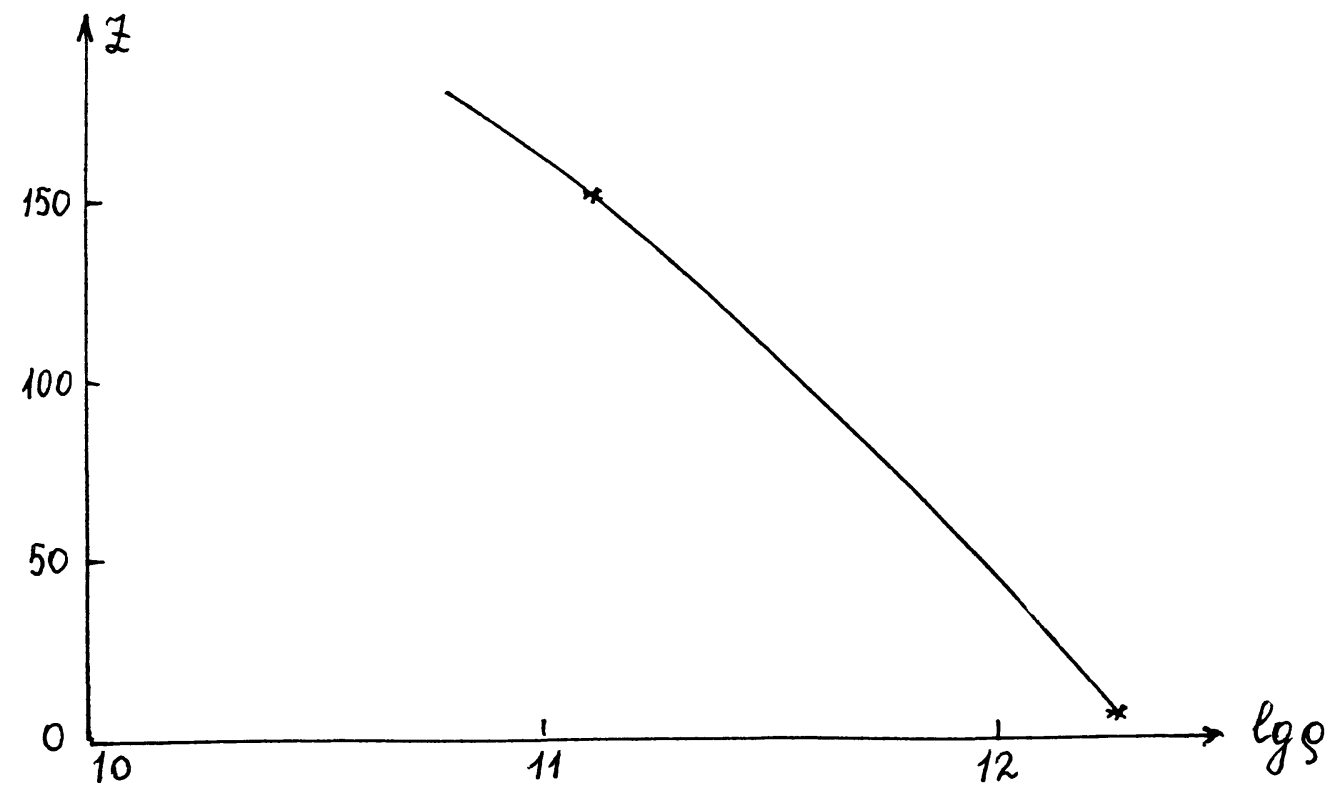

Fig. 1. Composition of neutron star envelope after cooling for $X_{n}=1 / 2$, nonequilibrium layer is situated at densities in between the asterisks

Note that the nonequilibrium pressure is $\sim 1.5$ times higher than the equilibrium one at the same density. The total mass and energy stored in this layer are [16]: 


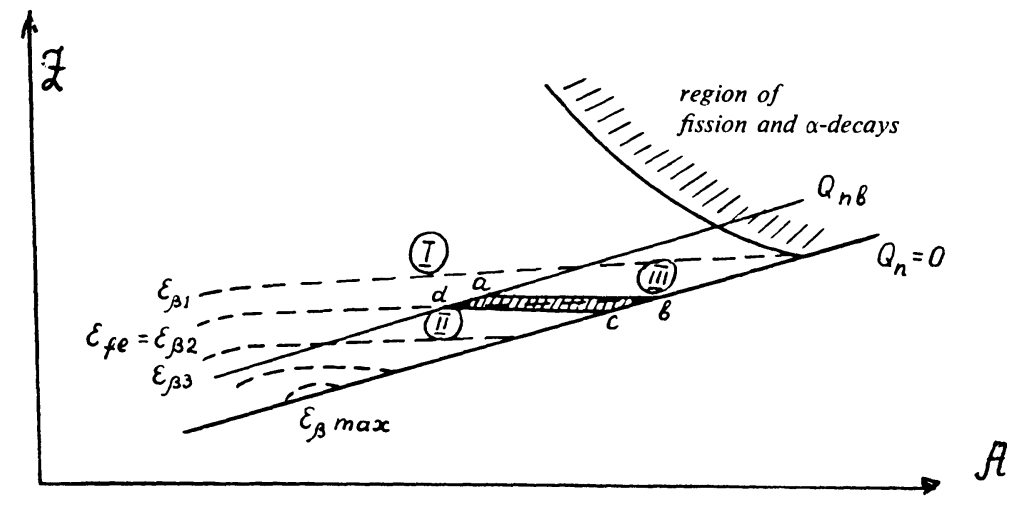

Fig. 2. Formation of non-equilibrium composition; photostripping of neutrons is impossible in region $I$, where $Q_{n}>Q_{n b}$; in region II $-Q_{n}<Q_{n b}, \varepsilon_{f e}<\varepsilon_{\beta}$, and in region III $-Q_{n}<Q_{n b}, \varepsilon_{f e}>\varepsilon_{\beta}$. On the dash lines $\varepsilon_{\beta}=Q_{p}-Q_{n}=$ const, $\varepsilon_{\beta 1}<\varepsilon_{\beta 2}<\varepsilon_{\beta \max }$. Nuclei are situated within the shaded region abcd at given values of $Q_{n b}(T)$ and $\varepsilon_{f e}(\rho)$

$$
m_{\text {tot }} \simeq 10^{29} \mathrm{~g}, \mathrm{E}_{\text {tot }} \simeq 3 \cdot 10^{47} \text { ergs } \text { for } \eta=3 \cdot 10^{-3} \mathrm{c}^{2} .
$$

3. NUCLEAR EXPLOSION IN THE OUTER LAYERS OF THE NEUTRON STAR ENVEIOPE

A very sharp increase of fuminosity observed in the 5 March 1979 burst $\left(\tau_{r}<2 \cdot 5 \cdot 10^{-4}\right.$ s [21] $)$ is incompatible with

a thermonuclear explosion of the accreted material, where the rise times $0.2-1 \mathrm{~s}$ and the spectrum explain well the properties of much softer X-ray bursters identified with neutron stars in low-mass binary systems [22]. The nuclear fission beneath the surface of the neutron star leads to an explosion due to the chain reaction to formmation of the shoch wave and very sharp increase of luminosity in good accordance with observations.
a) Development of
a chain reaction
[13]

Let us suppose that during a starquake the matter from the layer at $\rho_{1}<\rho<\rho_{2}$ is transported to a lower-density re- 
gion $\rho \ll \rho_{1}$ with the depth $d \ll 10^{4} \mathrm{~cm}{ }^{9}$ ). We now assume for simplicity, that initially the matter consists of the kind of nuclei (A,Z) which are stable against fission and $\alpha$-decay, but not against $\beta^{-}$-decay at $\rho \ll 10^{10} \mathrm{~g} / \mathrm{cm}^{3}$. The interaction of these nuclei with rapid neutrons lead to the formation of slow neutrons, so these nuclef act as a moderator material. This property of the nuclei ( $A, Z)$ follows from the fact that the nonequilibrium layer at $\rho_{1}<\rho<\rho_{2}$ is stable even in the presence of chance rapid neutrons. The nucleus $(A, Z)$ takes part in two reactions:

$$
\begin{aligned}
& (A, Z) \longrightarrow(A, Z+1)+e^{-}+\tilde{z}, \\
& (A, Z)+n_{1} \longrightarrow(A, Z)+n_{s},
\end{aligned}
$$

where $n_{r}$ and $n_{s}$ are rapid and slow neutrons. Nuclei $(A, Z+1)$ are stable to the spontaneous fission and $\alpha-d e-$ cay, but unstable to $\beta^{-}$-decay and the fission induced by rapid neutrons. So we have

$$
\begin{aligned}
& (A, Z+1) \longrightarrow(A, Z+2)+e^{-}+\tilde{\nu}, \\
& (A, Z+1)+n_{r} \longrightarrow\left(A_{1} Z_{1}\right)+\left(A_{2} Z_{2}\right)+(\alpha+1) n_{r} .
\end{aligned}
$$

The nucleus $(A, Z+2)$ is unstable to $\beta^{-}$-decay and spontaneous fission and yields $\zeta$ rapid neutrons in one fis-
sion:

$$
\begin{aligned}
& (A, Z+2) \longrightarrow(A, Z+3)+e^{-}+\tilde{\nu}, \\
& (A, Z+2) \longrightarrow\left(A_{3}, Z_{3}\right)+\left(A_{4}, Z_{4}\right)+\zeta n_{r} .
\end{aligned}
$$

The picture of the development of explosion is. as follows. When bulk of the matter consisting of nucle 3 ( $\mathrm{A}, \mathrm{Z}$ ) comes to a region with low density $\rho \ll 10^{10} \mathrm{~g} / \mathrm{cm}^{3}$, the $\beta^{-}$

decay begins, its prabability being $W_{\beta}^{z}$. When a sufficient amount of nuclei $(A, Z+1)$ is stored, the absorption of rapid neutrons by the moderator, characterized by the average cross-section multiplied by velocity $\langle\sigma \mathrm{V}\rangle$, becomes less intense. The production of rapid neutrong in *) This transportation does not need energy supply because the entropy of a neutron star is close to constant (zero) which mean that there exists neutral equilibrium to convective (exchange) motion. 
the induced fission ( 8$)$ of $(A, Z+1)$ characterized by $\langle\sigma V\rangle_{z+1}$ and spontaneous fission (9) of $(A, Z+2)$ which follows immediatly the $\bar{\beta}$-decay of $(A, Z+1)$ with probability $w^{\mathrm{z}+1}$, prevails as compared with their absorption, a chain reaction develops and a nuclear exposion occurs. For approximate values $[13]\left(\mathrm{V}_{\mathrm{n}} \simeq 3.10^{9} \mathrm{~cm} \mathrm{~s}^{-1}\right.$ for $\mathrm{E}_{\mathrm{n}_{\mathrm{r}}} \simeq$
$10 \mathrm{MeV})$ :

$$
\begin{aligned}
& w_{\beta}^{z} \simeq 10^{3} \mathrm{~s}^{-1}, \mathrm{w}_{\beta}^{\mathrm{z}+1} \simeq 10^{2} \mathrm{~s}^{-1}, \\
& \langle\sigma \nabla\rangle_{\mathrm{z}} \simeq 10^{-15} \mathrm{~cm}^{3} \mathrm{~s}^{-1},\left\langle\sigma v_{\mathrm{z}+1} \simeq \frac{1}{3} 10^{-17} \mathrm{~cm}^{3} \mathrm{~s}^{-1}\right. \\
& \alpha=\gamma \simeq 3
\end{aligned}
$$

the chain reaction begins when

$$
\frac{x_{z+1}}{x_{z}} \simeq \frac{\langle\sigma v\rangle_{z}}{\alpha\langle\sigma v\rangle_{z+1}} \simeq 100
$$

and concentration of rapid neutrons $X_{r}$ is

$$
x_{r}=\frac{\gamma \cdot w_{\beta}^{Z+1}}{\left(\rho / m_{p}\right) \alpha\langle\sigma v\rangle_{z+1}} \simeq 5.10^{-15} \text { for } \rho=10^{6} \mathrm{~g} \cdot \mathrm{cm}^{-3}
$$

Chain reaction begins

$$
\frac{\ln 100}{\mathrm{w}_{\beta}^{\mathrm{Z}}} \simeq 5 \cdot 10^{-3} \mathrm{~s}
$$

after the starquake and develops practically instantly, so that

$$
\tau_{\mathrm{ch}} \ll \tau_{\mathrm{h}} \simeq \frac{\mathrm{d}}{v_{\mathrm{s}}} \frac{10^{4}}{10^{10}}=10^{-6} \mathrm{~s}
$$

The average energy output (essiciency) in the fission of superheavy nuclei may be estimated [15] as $\eta=$ $=3.10^{-3} \mathrm{c}^{2} \mathrm{ergs} / \mathrm{g}$. Instant heating leads to the formation of a shock wave which may initiate gamma ray burst when it reaches the suriace. 
b) Formation and output of the shock wave

Hydrodynamical calculations of the shock wave propagation in the neutron star envelope have been done in [14] in $a$ spherically symmetric approximation. The system of equations in Lagrangian coordinates is:

$$
\begin{aligned}
& \frac{\partial r}{\partial t}=u, \quad \frac{\partial r}{\partial m}=\frac{1}{4 \pi \rho r^{2}}, \\
& \frac{\partial u}{\partial t}=-4 \pi r^{2} \frac{\partial p}{\partial m}-\frac{G m}{r^{2}}, \\
& \frac{\partial \varepsilon}{\partial t}=-4 \pi p \frac{\partial\left(u r^{2}\right)}{\partial m}+q(m, t)-f_{\nu}(\rho, \tau) .
\end{aligned}
$$

An approximate equation of state in the analytical form [23] has been used:

$$
\begin{aligned}
P & =3.09 \cdot 10^{12} \rho^{5 / 3} \frac{1+1.59 \cdot 10^{-3} \rho^{1 / 3}}{\left(1+3.18 \cdot 10^{-3} \rho^{1 / 3}\right)^{2}}+\rho R T+ \\
& +\frac{a T^{4}}{3} d y \cdot n / \mathrm{cm}^{2}, \\
\varepsilon & =4.635 \cdot 10^{12} \rho^{2 / 3}\left(1+3.18 \cdot 10^{-3} \rho^{1 / 3}\right)^{-1}+\frac{3}{2} R T+ \\
& +\frac{a T^{4}}{3} \mathrm{ergs} / g,
\end{aligned}
$$

which is valid for $\rho \leqslant 3 \cdot 10^{9} \mathrm{~g} / \mathrm{cm}^{3}, Q=k_{B} / \mu m_{p}$ - gas constant, $\mu=1$, a - energy density constant. The neutrino emission rate $f_{\nu}(\rho, T)$ takes into account URCA processes and interpolates numerical calculations from [24].

Only the region with $\rho<3.109 \mathrm{~g} / \mathrm{cm}^{3}$ has been considered in calculations. The star is supposed to be initialIy cold and in static equilibrium:

$$
u=0, \frac{\partial u}{\partial t}=0, T=0 \text { at } t=0 \text {. }
$$

The explosion was characterized by the energy $F_{t}$, which was produced very rapidiy $t_{p} \ll t$ in a sphericapt
layer whose upper edge is at a depth $h$ under the surface. The mass of this layer $m_{m}$ and the depth of its bottom $h_{1}$ are determined uniqhely by the efficiency $\eta$ : $\mathrm{m}_{\mathrm{b}}=\mathrm{E}_{\text {tot }} / \mathrm{q}$. So, in this layer 


$$
\begin{array}{rlrl}
q(m, t) & =\frac{\eta}{t_{b}}, & 0 \leqslant t \leqslant t_{b}, \\
& =0 & t>t_{b},
\end{array}
$$

with $t_{b}=10^{-8} \mathrm{~s}$. At $t=t_{b}$ the temperature distribution is determined by using (16):

$$
\frac{3}{2} R \mathrm{~T}_{\mathrm{b}}+\mathrm{a} \mathrm{T}_{\mathrm{b}}^{4} / \rho=\eta \text {. }
$$

Typical distributions of parameters at $t=0$ and $t=t_{b}$ are given in Fig. 3. The results of numerical calculations
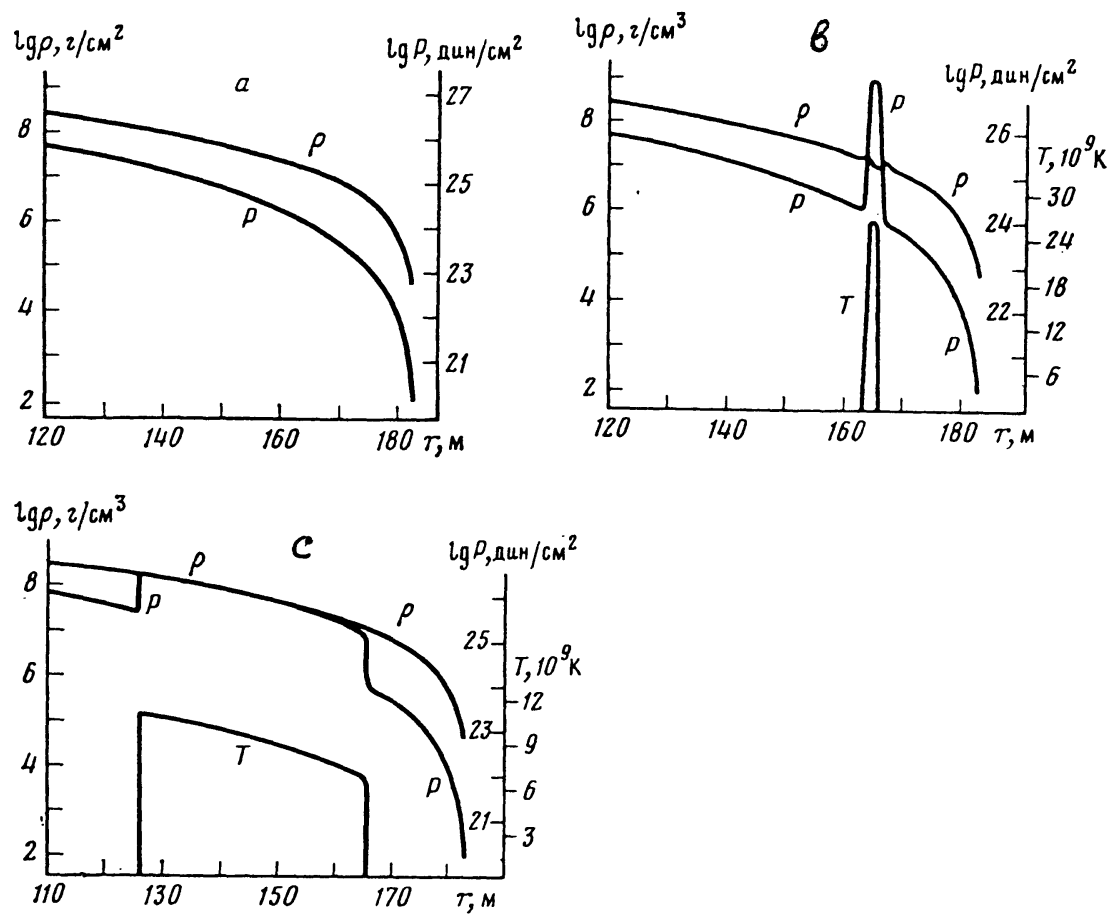

Fig. 3. Density, pressure and temperature distributions in the envelope of the neutron star with $\mathbb{M}=1 \mathrm{M}_{\odot}$, $R=10 \mathrm{~km}$. The distance $r_{3}$ is measured from the levei $\rho=3 \cdot 109 \mathrm{~g} / \mathrm{cm}^{3}$. a) $t=0$ with $\mathrm{T}=0$, b) $t=t_{b}$, variant 1 from table $\left.1, c\right) t=t_{b}$, variant 6 from table 1 .

are presented in Table 1 and Fig. 4. The surface temperature at the moment when the shock wave reaches the surface with optical depth $\tau=1$ was estimated in [14], using 
Table 1. Explosion parameters in the envelope of the neutron star with $M=1 M_{\odot}, R=10 \mathrm{~km}$

\begin{tabular}{|c|c|c|c|c|c|c|}
\hline IN & $\eta / c^{2}$ & $\begin{array}{l}E_{\text {tot }} \\
\text { ergs }\end{array}$ & $\begin{array}{l}h_{0}-h \\
\text { meters }\end{array}$ & $\mathrm{m}_{\mathrm{b}} / \mathrm{M}_{\theta}$ & $\begin{array}{c}\mathrm{M}_{\mathrm{hyd}} / \mathrm{M}_{\mathrm{O}} \\
\left(\begin{array}{ll}\% & \left.\mathrm{~m}_{\mathrm{b}}\right)\end{array}\right.\end{array}$ & $\frac{u_{\text {edge }}}{u_{p}}$ \\
\hline 1 & 0.3 & $10^{43}$ & $18-20$ & $1.86 \cdot 10^{-11}$ & $\begin{array}{c}8.7 \cdot 10^{-13} \\
(4.7)\end{array}$ & $>1$ \\
\hline 2 & 0.03 & $10^{43}$ & $18-30$ & $1.86 \cdot 10^{-10}$ & $\begin{array}{r}3.0 \cdot 10^{-14} \\
(0.016)\end{array}$ & $>1$ \\
\hline 3 & 0.008 & $10^{43}$ & $18-40$ & $6.94-10^{-10}$ & $\left(\leqslant 10^{-3}\right)$ & 0.64 \\
\hline 4 & 0.008 & $10^{43}$ & $40-50$ & $6.94 \cdot 10^{-10}$ & $\left(\leqslant 10^{-3}\right)$ & 0.86 \\
\hline 5 & 0.003 & $10^{42}$ & $10-30$ & $1.86 \cdot 10^{-10}$ & $\left(\leqslant 10^{-}\right.$ & 0.34 \\
\hline 6 & 0.003 & $10^{43}$ & $18-58$ & $1.86 \cdot 10^{-9}$ & $\left(\leqslant 10^{-5}\right)$ & 0.34 \\
\hline 7 & 0.003 & $10^{43}$ & $68-76$ & $1.86 \cdot 10^{-9}$ & $\left(\leq 10^{-5}\right)$ & 0.61 \\
\hline 8 & 0.003 & $10^{44}$ & $20-174$ & $1.86 \cdot 10^{-8}$ & $\left(\leqslant 10^{-5}\right)$ & 0.36 \\
\hline
\end{tabular}

selfsimilar solution by the method, proposed in [5]

$$
p \simeq k_{2}^{2} \rho h^{-0.6}, \rho \simeq k_{1} h^{1.5}, u=0,7 k_{2} h^{-0.3} .
$$

Constants $k_{2}$ and $k_{1}$ are estimated from numerical calculations. ${ }^{2}$ Applying (20) at $\tau=\int_{0}^{h} x \rho d h=1$, where numerical calculations failed, we obtain the surface temperature $T_{1}=150 \mathrm{KeV}$ for models in Table 1 with $\eta=$ $=0.003$. This surface temperature leads to supercritical luminosity and strong mass loss. Supposing that the momentum of radiation is transformed into the momentum of matter running away with velocity close to parabolic one $u_{p}=(2 G M / R)^{1 / 2} \simeq 0.54 c$, we obtain for the total mass loss nout :

$$
m_{\text {out }} \simeq \frac{\alpha E_{\text {tot }}}{0.54 c^{2}} \simeq 2 \cdot 10^{22} \alpha\left(\mathrm{F}_{\text {tot }} / 10^{43}\right)
$$

where $\alpha=0.5-1$ is part of the energy emitted in the su- 

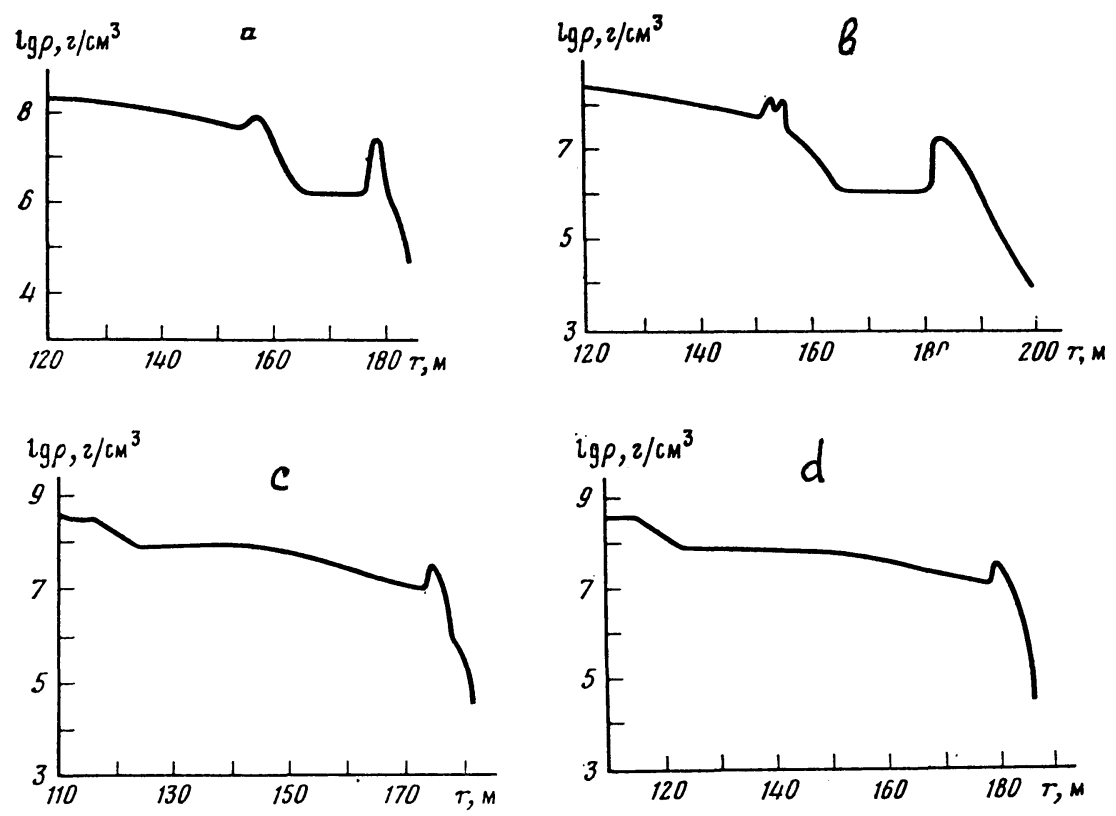

Fig. 4. Density distribution at different moments of the development of the explosion in the star with $M=1 M_{\odot}, R=10 \mathrm{~km}$. Variant 1 from table $\left.1: a\right) t=20 t_{b}-$ before the output of the shock wave to the surface; b) $t=30 t_{b}$ - after the output. Variant 6 from table 1: c) $b_{t}=130 t_{b}$ - before the output; d) $t=160 t_{b}$ after the output

percritical regime. For $\eta=0.003 c^{2}$ the part of the running-away matter is equal to $\sim 0.007 \alpha$ of the exploded one. Note that in real events the diameter of the bulk of exploding matter is equal about its depth $h$, so the numbers for energy in Table 1 should be multiplied by

$$
\frac{h^{2}}{4 \pi R^{2}} \simeq 10^{9} /\left(4 \pi \cdot 10^{12}\right) \simeq 10^{-4}
$$

for $h=3 \cdot 10^{4} \mathrm{~cm}$ and $\mathrm{R}=10 \mathrm{~km}$. The amount of mass ejected by the supercritical wind from (21) is much larger than a hydrodynamical outburst $m_{h y d}$ by the shoch wave in Table 1.

4. MODEL OF THE GAMMA RAY BURST 5 MARCH 1979

The observed flux $Q_{\gamma}=1 \cdot 3 \cdot 10^{-3} \mathrm{ergs} / \mathrm{cm}^{2}$ from this sour- 
ce leads to the following total energy outputs for different assumed distances $d_{\text {LMC }}$ and $d_{G a I}$ :

$$
\begin{aligned}
& q_{\text {tot }}=4 \cdot 5 \cdot 10^{44} \text { ergs for } d_{\text {LMC }}=55 \mathrm{kpc} \\
& q_{\text {tot }}=1 \cdot 5 \cdot 10^{39} \mathrm{ergs} \text { for } d_{\text {GaI }}=100 \mathrm{pc} .
\end{aligned}
$$

The observed luminosity the pulsar stage is

$$
\begin{aligned}
& I_{\text {puls, LMC }}=3 \cdot 6 \cdot 10^{42} \mathrm{ergs} / \mathrm{s} \gg I_{\text {edd }}, \\
& I_{\text {puls, Gal }}=1.2 \cdot 10^{37} \mathrm{ergs} / \mathrm{s}<I_{\text {edd }} .
\end{aligned}
$$

The rotational energy of the neutron star with the observed period $p=8.1 \mathrm{~s}$ is

$$
E_{\text {rot }} \leqslant \frac{1}{2} I_{\max } \Omega^{2} \simeq 5 \cdot 10^{44} \text { ergs } \simeq q_{\text {tot } L M C} .
$$

Having in mind that not more than $0.02 \mathrm{E}_{\text {rot }}$ (energy of the crust) may be releated during a starquaket we may exclude the possibility of rotational energy supply in the case of LMC. The explosion of the nonequilibrium matter of such a scale seems to be also improbable. The observed behavior of the burst source on the pulsar stage is rather regular $[3,21]$ and cannot be related with strongly supercritical regime, where violent outbursts of matter and strong irregularities of luminosity must be expected. So we adopt the galactical origin of this burst.

The exist-ance of spectral features in this burst indicates that optical depth $\tau \leqslant 1$ during their formation. We suppose $[4,10]$ that the spectrum of a $\gamma$-ray burst is a mixture of the emission of a hot spot on the surface of the neutron star and of an expanding cloud which becomes transparent to $\gamma$-radiation at $t \geqslant t e_{\text {For }}$ the cloud expanding with velocity $V_{c} \simeq 0.5 \&$ and supposing, we estimate cloud parameters as?

$$
\begin{aligned}
\tau_{c}<1 \text { at } t=t_{0} & =1.5 \cdot 10^{9}\left(\frac{t_{o}}{0.1 \mathrm{c}}\right), \\
R_{c} & \simeq V_{c} t_{0} \simeq 1 . \\
\rho_{c} & \simeq \frac{\mu_{e} m_{p}}{R_{c} \sigma_{e}} \tau_{c} \simeq \frac{10^{-8}}{t_{0.1}} \tau_{c} \mathrm{~g} / \mathrm{cm}^{3},
\end{aligned}
$$




$$
\begin{aligned}
& M_{c}=\frac{4 \pi}{3} \rho_{c} R_{c}^{3}=1.4 \cdot 10^{20} t_{0.1}^{2} \tau_{c} g, \\
& t_{0.1}=t_{0} / 0.1 \mathrm{sec}
\end{aligned}
$$

Here we used $\sigma_{e}=0.4 \sigma_{T} \simeq 2.6 .10^{-25} \mathrm{~cm}^{2}$ for $E_{\gamma} \simeq$ $0.6 \mathrm{MeV}$ and $\mu_{e}=2.3$. If we suppose that nonthermal emission is generated in the expanding cloud with efficiency $\frac{1}{4} \eta=\frac{1}{4} 3 \cdot 10^{-3}$, then we obtain:

$$
E_{\text {nonth }}=\frac{3 \cdot 10^{-3}}{4} M_{c} c^{2}=10^{38}\left(t_{0.1}\right)^{2} \tau_{c} \text { ergs }
$$

The total energy of the burst is about two orders of magnitude larger and is equal to

$$
E_{\text {tot }} \simeq 10^{40} t_{0.1}^{2} \tau_{c} \text { ergs }
$$

For the integral flux on the Earth $F_{c} \simeq 1.3 \cdot 10^{-3} \mathrm{ergs} / \mathrm{cm}^{2}$, we estimate the distance as

$$
d=\left(E_{\text {tot }} / 4 \pi \mathrm{F}_{\mathrm{c}}\right)^{1 / 2} \simeq 8.10^{20} \mathrm{t}_{0.1} \tau_{\mathrm{c}_{\mathrm{cm}}}^{1 / 2} \simeq 260 \mathrm{t}_{0.1} \tau_{\mathrm{c}_{\mathrm{pc}}}^{1 / 2}
$$

On the pulsar stage the spectum is well approximated by the radiation of optically thin plasma with $\mathrm{T}=4 \cdot 10^{8} \mathrm{~K}$

[3], so that the emission region should be represented by a corona on the surface of the neutron star. The existence of a corona may be connected with the mechanical flux from a convective layer in the stellar envelope developed after the explosion, and the magnetic field may be important for mechanical energy transformation into heat, like in the heating of the solar corona.

Let us estimate coronal parameters $[11,12]$. The density distribution in the isothermal corona is represented by a barometric formula

$$
\rho=\rho_{0} e^{-\left(\mu_{\mathrm{p}} / k T\right)\left(G M / R_{n s}^{2}\right) x}=\rho_{0} e^{-(X / 113)} .
$$

The flux of free-free emission from the unit surface is 
equal to

$$
\begin{aligned}
& F_{\text {cor }}=\int_{0}^{\infty} 1.4 \cdot 10^{-27} z^{2} \sqrt{T_{c}} n_{e^{n_{i}}} d x=8 \cdot 10^{-22} z_{n_{e}}^{2} \\
& \mathrm{n}_{e}=\mathrm{Zn} \mathrm{n}_{\mathrm{i}} \quad \mathrm{ergs} / \mathrm{cm}^{2} \mathrm{~s}
\end{aligned}
$$

The value of $F_{\text {cor }}$ must be less than a critical Eddington flux $F_{\text {Edd }}$, so

$$
\begin{aligned}
& \mathrm{F}_{\text {cor }}=8 \cdot 10^{-22} \mathrm{Zn}_{\mathrm{eo}}^{2}=\varepsilon \mathrm{F}_{\mathrm{edd}}=\varepsilon 4 \cdot 10^{24} \frac{\mu_{\mathrm{e}} \mathrm{m}_{\mathrm{p}}}{\sigma_{T}}, \\
& \varepsilon<1, \mathrm{n}_{\mathrm{eo}}=2.8 \cdot 10^{22} \sqrt{\varepsilon}, \mu_{e}=2.3 .
\end{aligned}
$$

We take $\varepsilon \leqslant 0.1$ so that when compton corrections to the spectrum are neglible [11]. On the pulgar stage the flux on the Earth $I_{e}$ is equal to $1_{e}=10^{-5} \mathrm{ergs} / \mathrm{cm}^{2} \mathrm{~s}$. If loalf

aboutvthe neutron star surface is heated, then for $\mathcal{E}=$ $=0.07, \mu_{e}=2.3$ we have from (31):

$$
\begin{aligned}
I_{e} & =\frac{1}{2}\left(\frac{R_{n s}}{d}\right)^{2} F_{c o r}, d=\left(\frac{F_{\text {cor }}}{2 I_{e}}\right)^{1 / 2} R_{n s}= \\
& =2.6 \cdot 10^{20} \mathrm{~cm}=85 \mathrm{pc} .
\end{aligned}
$$

Comparing (32) with (28) and (27) we have

$$
\begin{aligned}
& t_{0.1} \tau_{c}^{1 / 2} \simeq 1 / 3, E_{\text {tot }} \simeq 10^{39} \text { ergs, } \\
& \tau_{c}<1 \Rightarrow t_{0}>0.033, \\
& t_{0}<0.2 \mathrm{~s} \Rightarrow \tau_{c}>0.02 .
\end{aligned}
$$

The value of $E_{\text {tot }}$ is much lessyantotal energy reserve of nonequilibrium layer in (6). The qualitative picture of the development of this burst is shown in Fig. 5 . 

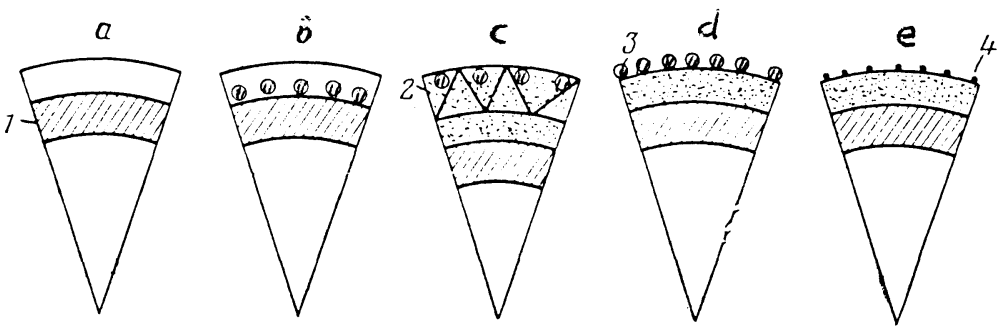

Fig. 5. Schematic picture of gamma-ray burst formation 5 March 1979; a - envelope in static equilibrium, 1 - nonequilibrium layer; b - after starquake, c after nuclear explosion, 2 - shock wave, d - expansion of the cloud ( 3 ) and explosions there, e pulsar stage, 4 - hot corona

5. SPECTRAL FEATURES IN THE EMISSION OF GAMMA-RAY BURSTS

Several kinds of features have been observed in the spectra of gamma-ray bursts. The first are emission features in the hard region which are broad $\triangle \mathrm{E} \simeq \mathrm{E}$ and have maxima at $\mathrm{E} \simeq 400-500 \mathrm{KeV}$, close to the $\mathrm{e}^{+} \mathrm{e}^{-}$- annihilation Iine [25] It was supposed in [25], that these features are emitted in optically thin not regions, separated from the regions which emit most of their energy in softer photons. In our model this region may be an expanding cloud or more probabIy a corona on the initial stage of the burst, when its temperature may be sufficiently high for intense thermal birth of $e^{+} e^{-}$- pairs.

The second type of features has been observed in a softer region $E_{r}=30-60 \mathrm{KeV}$ mostly in absorption [26] and has been interpreted there as cyclotron absorption. It seems improbable for an old neytron star to have very high magnetic field $3.1012-10^{13} \mathrm{Gs}$, nesessary for cyclotron spectral features to form in this region. Besides, such a large field prevents output of hard photons with $\mathrm{E} \gamma \geqslant 2 \mathrm{MeV}$ observed in the spectra of bursts. In our model these features may be naturally interpreted as absorption lines of very heavy elements with $\mathrm{Z} \simeq 55$ in an expanding cloud. These elements are produced in fission of superheavy nuclei. For example, $\mathrm{Ba}_{56}^{40}, \mathrm{I}_{53}$ with $\mathrm{A}=$ $=131-135$ and $\mathrm{Ce}_{58}$ with $\mathrm{A}=143,144$ are the main elements produced in fission of $U^{235}$ [27]. The ionization potential of the last $K$-electron $I_{Z} \simeq 13.6 \mathrm{Z}^{2} \mathrm{eV} \simeq$ $42.6 \mathrm{eV}$ for $\mathrm{Ba}_{56^{\circ}}$. In the cloud expanding with $\mathrm{V}=0.4 \mathrm{c}$ 
the blue shifted absorption line may be located close to the observed one at $60 \mathrm{KeV}$. Temporal behaviour of this absorption feature in some bursts [25] which gradually dissapeared is well explained by the absorption in the expanding cloud, which should disappear when the density in the cloud becomes small. If iron $\mathrm{Fe}^{56}$ is the most abumdant element in the cloud, then we may expect in some cases strong absorption in the softer region $\mathrm{E} \geqslant 13 \mathrm{KeV}$ during the cloud expansion.

In the hard region $E \geqslant 1 \mathrm{MeV}$ spectral features may be expected which are associated with the emission of excited nuclei. Most probable is $\mathrm{D}^{2}$ line with $\mathrm{E}_{\mathrm{r}}=2.2 \mathrm{MeV}$. Such a line may be formed in our model, bedause free neutrons are formed during the fission of superheavy nuclei [10]. Indications to the presence of this line have been found in the spectra of the 7 March 1979 burst in some spectra obtained over short periods $\Delta t \leqslant 1$ sec in the Soviet-French signe experiment on Venera 11 and 12 probes.

\section{OPTICAL EMISSION ACCOMPANYING GAMMA BURST}

Intense investigations of the error-box of the 5 March 1979 gamma-ray burst lead to a discovery of optical flashes which may be connected with gamma ray bursts [28]. The shape of one of these flashes on $8 \mathrm{Feb} .1984$ is close to that of the main burst (Fig. 6). Several weaker recurrent gamma events have been observed in the same direction [29] The optical flash may be associated with one of the recurrent rather feeble gamma-ray bursts.

The shape of this optical flash is well interpreted by the optical emission of the expanding cloud. The emission prior to the maximum (Fig. 6) may be connected with a Reyleigh-Jeans region of thermal emission of the cloud which is opaque in the optical region. The optical emission after the maximum may be interpreted as free-free radiation of optically thin iron plasma cloud, irradiated by the gamma-ray emission of the neutron star. Estimations made together with A.F. Illarionov, show that optical emission of the transparant oloud may be about

$5 \cdot 10^{-4}$ times the gamma luminosity of the neutron star.

\section{REFERENCES}

1. Klebesadel R•, Strong I., Olson R., Astrophys J. Lett., 1973, 182, L85. 


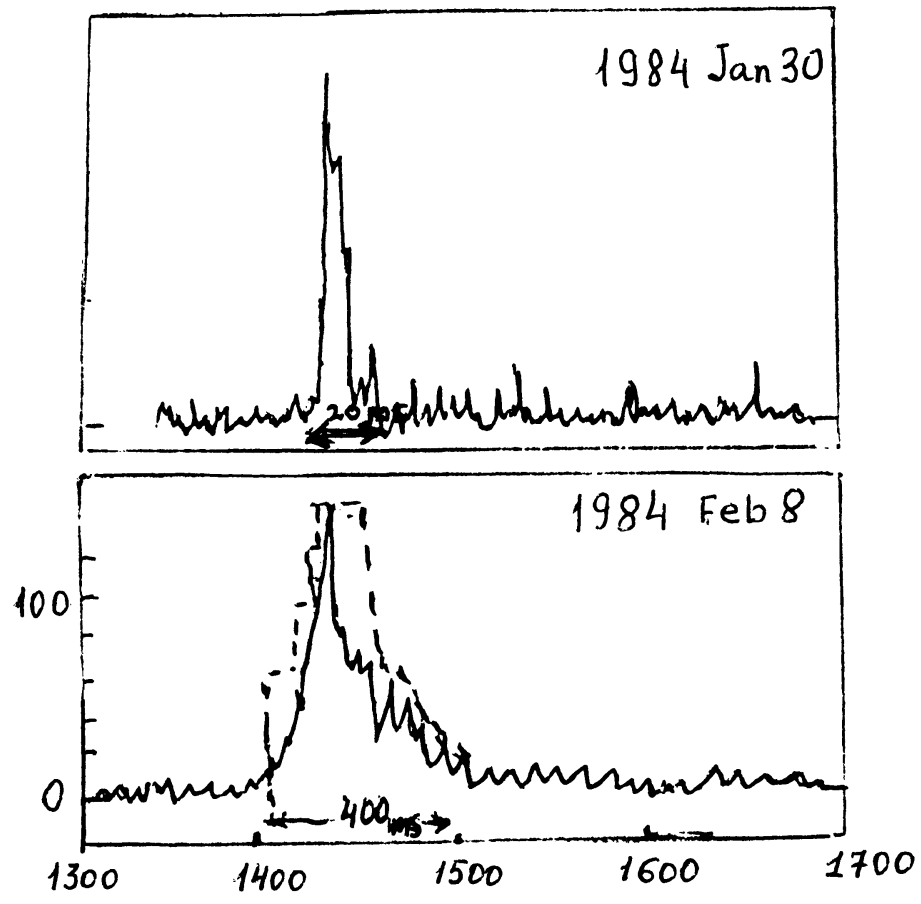

Fig. 6. Optical flash $8 \mathrm{Feb} .1984$ and gamma-ray burst 5 March 1979 coming from the same region of the sky. The times of beginning of both flashes are brought into coincide as well as their maxima due to choosen luminocity scales

2. Prilutskii O.F., Rozental I.I., Usov V.V., Uspechi Fiz. Nauk, 1975, 116, 517.

3. Mazets F.P., Golenetskii S.V., Ilyinskii K.N., Panov V.N., Aptekar R.I., Gurjan Yu.A., Sokolova Z.Ya., Charitonova T.V., Pisma Astron. Zh., 1979, 5, 307.

4. Bisnovatyi-Kogan G.S., Gamma-Ray bursts from neutron stars, Report on COSPAR/IAU Symposium on "Fast Transients in X and gamma-ray astronomy", May 29-31, 1975, Varna, Bulgaria; Space Res. Inst. preprint D-203,1975.

5. Bisnovatyi-Kogan G.S., Imshennik V.S., Nadyozhin D.K., Chechetkin V.M. Astrophys. Space Sci., 1975, 35, 23,

6. Mazets E.P. et al. Catalog of cosmic gamma ray bursts from the Konus experiment data. Astrophys. Space Sci., 1981, vol. $80, \mathrm{~N} 1$.

7. Vedrenne G. Cosmic $\gamma$-ray bursts, Phil. Trans. R. Soc. Lond. 1981, A301, 645.

8. Hurley K., Adv. Space Res., 1983, 3, 163.

9. Rozental I.I., Usov V.V., Estulin I.V., Uspechi Fiz. Na uk, 1983, 140, 97.

10.Bisnovatyi-Kogan G.S., Chechetkin V.M. Sov. Phys. Usp., 1979, 2․, 89. 
11. Bisnovatyi-Kogan G.S., Chechetkin V.M. Astron. Zh. USSR, $1981,58,561$.

12. Bisnovatyi-Kogan G.S., Chechetkin V.M. Adv. Space Res. $1981,1,153$.

13. Bisnovātyi-Kogan G.S., Chechetkin V.M. Astrophys. Space Sci., 1983, 89, 447 .

14. Bisnovatyi-Kogan G.S., Blinnikov S.I., Zacharov A.F. Astron. Zh. USSR, 1984, 61, 104.

15. Bisnovatyi-Kogan G.S., Chechetkin V.M. ZhTF, Pisma, 1973, 17, 622.

16. Bisnovatyi-Kogan G.S., Chechetkin V.M. Astrophys. Space Sci., 1974, 26, 25.

17. Vartanyan Yu.L., Ovakimova N.K. Soobsch . Burakan. Obs. 1976, N 49, p. 87 .

18. Sato K. Prog. Theor. Phys. 1979, 62, 957.

19. Grootez H.V., Hilf $\mathrm{E}_{\bullet} \mathrm{R}_{\bullet}$, Takahashi $\mathrm{K}_{\bullet}, \mathrm{Af}$. data and nucl. data tables, 1976, 17, 418-427, 476-608.

20. Baym G., Bethe H., PethickGh. Nucl. Phys., 1971, A175, 225.

21. Cline T. et al. Astrophys. J. Let. 1980, 232, I1.

22. Joss P., Li F. Astrophys. J., 1980, 238 , 287.

23. Bisnovatyi-Kogan G.S., Popov Yu.P., Samochin A.A. Astrophys. Space Sci., 1976, 41, 287.

24. Ivanova I.N., Imshennik V.S., Nadyozhin D.K. Nauch. Inf. Astron. Sov. Acad. Sci. USSR, 1969, 13, 3.

25. Golenetskii S.V. et al. Ioffe Phys.-Tech. Inst. preprint $\mathrm{N}$ 959, 1985.

26. Mazets F.P. et al. Ioffe Phys.-Tech. Inst., preprint $\mathrm{N} 687,1980$.

27. Shirokov Yu.M., Yudin N.P. Nuclear physics, 1980, Nauka, Moscow.

28. Pedersen H. et al. Nature, 1984, 312, 46.

\section{DISCUSSION}

E. Liang: You interpret the "cyclotron feature" as due to resonance lines of heavy elements I-Pb. Does this not require an unreasonably high concentration of heavy elements?

G. Bisnovatyi-Kogan: We need about $1 \%$ of heavy element to Fe ratio. This is much higher than solar abundance but may be obtainable in the expanding cloud after the fission chain reaction.

K. Hurley: How are the recurrent bursts froin the 5 March source generated in this model?

G. Bisnovatyi-Kogan: The recurrent bursts have been observed only from 5 March 1979 gamma-ray burst position and they are at least $10^{3}$ times weaker than it. Maybe, they are connected with residual activity of the neutron star after the strong starquake. 
S. Woosley: The weak interaction time scale does not set a limit to the thermonuclear model for $\gamma$-ray bursts because helium may burn on a very rapid timescale at high density and temperature. Fryxe11 and Woosely have shown that a detonation wave will propagate around a neutron star in about a millisecond.

G. Bisnovatyi-Rogan: The helium flashes could explain X-ray bursters and it seems improbable that such different phenomena are produced by the same burning. You need a larger magnetic field for your model, which is prohibited by the observed hard $\gamma$-ray photons $\left(E_{\gamma}>2-5 \mathrm{MeV}\right)$ which cannot escape from the neutron star with large field.

S. Woosley: What is the recurrence time scale for your model? What sets that time scale?

G. Bisnovatyi-Kogan: The recurrence time is expected to be between approximately 100-1000 years and is determined by the time between subsequent starquakes in old neutron stars.

D. Arnett: In core collapse we consider physical conditions very similar to these you quote: We estimate the relaxation time (to remove nonequilibrium) to be $2 \times 10^{-5}$ sec. There seems to be a serious disagreement.

G. Binovatyi-Kogan: We consider the stages after formation of the neutron star and subsequent cooling. The nonequilibrium layer is formed after the temperature decreases and become less than $\sim 2 \times 10^{9} \mathrm{~K}$, the time for the reactions between charged particles becomes very large, and the number of seed nuclei remains constant. At densities between $10^{11}-10^{12} \mathrm{~g} / \mathrm{cm}^{3}$ the larger nonequilibrium exists for a very long time.

A. Burrows: Do I understand you to mean that many radio pulsars and, in particular, the Crab and Vela should be $\gamma$-ray bursters?

G. Bisnovatyi-Kogan: $\mathrm{Crab}$ and Vela must give the $\gamma$-ray bursts, but they are very feeble because of their large distances.

A. Burrows: How can matter deep in the crust penetrate all the way to the surface? What is the dynamics?

G. Bisnovatyi-Kogan: The matter of an old neutron star is almost at constant entropy (very sma11) and so is close to neutral stability relative to convection. The elastic energy released in the starquakes may be enough for convective motion which transports matter from the nonequilibrium layer to smaller densities where it becomes unstable.

S. Kulkarni: I would like you to clarify some points. If I understand you correctly, we ought to see a $\gamma$ - and optical-burst from any neutron star when it glitches. So are you suggesting that is worthwhile comparing $\gamma$-ray burst catalogs and pulsar catalogs? In addition is it worthwhile cross-checking archival optical plates with radio pulsar positions?

G.S. Bisnovatyi-Kogan: That is right. But radio pulsars are too far away, so there is little chance of finding something. 